\title{
柱后衍生-高效液相色谱法测定玉米中伏马菌素 $\mathbf{B}_{1}$ 和 $\mathbf{B}_{2}$
}

\author{
张晓旭 ${ }^{1}$ ， 肖志勇 ${ }^{2}$ ，张红艳 ${ }^{3,4}$ ，杨丽丽 ${ }^{1}$ ，马丽艳 ${ }^{1,4 *}$
}

(1. 中国农业大学食品科学与营养工程学院, 北京 $100083 ; 2$. 北京市农业环境监测站, 北京 100029;

3. 中国农业大学理学院, 北京 $100193 ； 4$. 农业部农产品质量监督检验测试中心，北京 100083)

摘要: 建立了邻苯二甲醛 $(\mathrm{OPA})$ 柱后衍生-高效液相色谱测定玉米中伏马菌素 $\mathrm{B}_{1}$ 和 $\mathrm{B}_{2}\left(\mathrm{FB}_{1}\right.$ 和 $\left.\mathrm{FB}_{2}\right)$ 的方法。采用 ZORBAX SB- $\mathrm{C}_{18}$ 色谱柱, 以 $0.1 \mathrm{~mol} / \mathrm{L}$ 磷酸二氢钠溶液 $(\mathrm{pH} 3.3$ ) - 甲醇为流动相, 梯度洗脱。流动相流速为 0.8 $\mathrm{mL} / \mathrm{min}$, 柱温 $40{ }^{\circ} \mathrm{C}$; 衍生剂的流速为 $0.4 \mathrm{~mL} / \mathrm{min}$, 衍生温度为室温。实验对衍生剂缓冲液的 $\mathrm{pH}$ 、衍生剂的浓度和 流速、激发和发射波长等重要条件进行了优化。结果表明,衍生剂的 $\mathrm{pH}$ 在 10.5 、OPA 的质量浓度为 $2 \mathrm{~g} / \mathrm{L}$ 、流速为 $0.4 \mathrm{~mL} / \mathrm{min}$ 、激发波长 $335 \mathrm{~nm}$ 、发射波长 $440 \mathrm{~nm}$ 时测定效果良好, $\mathrm{FB}_{1} 、 \mathrm{FB}_{2}$ 在 $0.2 \sim 20 \mathrm{mg} / \mathrm{L}$ 范围内线性关系良 好, 相关系数大于 $0.999 ; \mathrm{FB}_{1}$ 和 $\mathrm{FB}_{2}$ 的检出限均为 $0.02 \mathrm{mg} / \mathrm{kg}$; 在 $0.1 \sim 4.0 \mathrm{mg} / \mathrm{kg}$ 范围内, 3 个添加水平的平均 回收率为 $82.5 \% \sim 89.8 \%$ 。该方法精确、简单、快速, 适合玉米中 $\mathrm{FB}_{1}$ 和 $\mathrm{FB}_{2}$ 的测定。

关键词:高效液相色谱;邻苯二甲醛;柱后衍生; 伏马菌素;玉米

中图分类号: O658 文献标识码:A 文章编号:1000-8713(2012)08-0792-06

\section{Determination of fumonisins $B_{1}$ and $B_{2}$ in corn by high performance liquid chromatography with post- column derivatization method}

ZHANG Xiaoxu ${ }^{1}$, XIAO Zhiyong ${ }^{2}$, ZHANG Hongyan ${ }^{3,4}$, YANG Lili ${ }^{1}$, MA Liyan ${ }^{1,4}$ *

(1. College of Food Science and Nutritional Engineering, China Agricultural University, Beijing 100083, China;

2. Beijing Agro-Environmental Monitoring Station, Beijing 100029, China; 3. College of Science,

China Agricultural University, Beijing 100193, China; 4. Supervision \&Testing Center

for Agricultural Products Quality, Ministry of Agriculture, Beijing 100083, China)

\begin{abstract}
A high performance liquid chromatography-fluorescence detection with post-column derivatization method was developed to detect fumonisin $\mathrm{B}_{1}\left(\mathrm{FB}_{1}\right)$ and fumonisin $\mathrm{B}_{2}\left(\mathrm{FB}_{2}\right)$ in corn. Several factors, such as the $\mathrm{pH}$ of derivatization buffer, concentration and flow rate of derivatization reagents, excitation wavelength, emission wavelength, which affected the detection of fumonisins were optimized. The separation was performed on a ZORBAX $\mathrm{SB} \mathrm{C}_{18}$ column operated at $40{ }^{\circ} \mathrm{C}$ with the gradient elution by two mobile phases of $0.1 \mathrm{~mol} / \mathrm{L}$ sodium dihydrogen phosphate solution $(\mathrm{pH} 3.3)$ and methanol at a flow rate of $0.8 \mathrm{~mL} / \mathrm{min}$. The derivatization was performed at ambient temperature. The $o$-phthalaldehyde (OPA) flow rate was 0.4 $\mathrm{mL} / \mathrm{min}$. The results showed that the optimum conditions were $\mathrm{pH} 10.5$ of the derivatization reagent, OPA concentration at $2 \mathrm{~g} / \mathrm{L}$, and excitation wavelength of $335 \mathrm{~nm}$, emission wavelength of $440 \mathrm{~nm}$. The linear plots of $\mathrm{FB}_{1}$ and $\mathrm{FB}_{2}$ were obtained between 0.2 to $20 \mathrm{mg} / \mathrm{L}$, with the correlation coefficients above 0.999 for both $\mathrm{FB}_{1}$ and $\mathrm{FB}_{2}$. The limits of detection of fumonisins $B_{1}$ and $B_{2}$ were $0.02 \mathrm{mg} / \mathrm{kg}$. The mean recoveries at the three spiked levels of $0.1-4.0$ $\mathrm{mg} / \mathrm{kg}$ were $82.5 \%-89.8 \%$. This method is accurate, simple, rapid and suitable for the determination of fumonisins $\mathrm{B}_{1}$ and $\mathrm{B}_{2}$ in corn.
\end{abstract}

Key words : high performance liquid chromatography (HPLC) ; o-phthalaldehyde (OPA) ; postcolumn derivatization; fumonisins; corn 
伏马菌素 (fumonisins) 是 1988 年南非 Gelderblom 等 ${ }^{[1]}$ 发现的一组结构相似的真菌毒素, 主要由 串珠镰刀菌 ( $F$. moniliforme)、多育镰刀菌 $(F$. proliferatum) 产生, 到目前为止已有 28 种伏马菌 素被分离、鉴定 ${ }^{[2]}$,其中以伏马菌素 $\mathrm{B}_{1}\left(\mathrm{FB}_{1}\right)$ 和伏 马菌素 $\mathrm{B}_{2}\left(\mathrm{FB}_{2}\right)$ 存在最广, 毒性最强。伏马菌素可 引起马脑白质软化症、猪的肺水肿、对老鼠的肝脏和 肾脏造成伤害、诱导癌症的发生,破坏家禽的免疫系 统等 ${ }^{[3-6]}$ 。伏马菌素对人类健康的影响目前尚不清 楚,但流行病学调查发现该毒素与人类食道癌、肺 癌、神经管畸形的发生率存在某种相关性 ${ }^{[7-9]}$ 。国 际癌症研究机构已将伏马菌素宣布为 “2B”级毒 物 ${ }^{[10]}$ 。2001 年 FAO/WHO ( Food and Agriculture Organization of the United Nations/World Health Organization) 食品添加剂联合专家委员会第 56 次 会议上,临时确立了人体每日允许摄人 $\mathrm{FB}_{1} 、 \mathrm{FB}_{2}$ 的 最大限量 (provisional maximum tolerable daily intake, PMTDI) 为 $2 \mu \mathrm{g} / \mathrm{kg}^{[11]}, 2007$ 年欧盟规定 供人直接食用的玉米及其制品中 $\mathrm{FB}_{1}$ 和 $\mathrm{FB}_{2}$ 总和 最高限量为 $1000 \mu \mathrm{g} / \mathrm{kg}$, 用于早餐和小吃的玉米制 品为 $800 \mu \mathrm{g} / \mathrm{kg}$, 加工的玉米制品及婴儿食品为 200 $\mu \mathrm{g} / \mathrm{kg}^{[12]}$ 。我国尚未制定食品中伏马菌素的限量 标准。因此,建立一种有效的检测方法开展谷物及 食品中伏马菌素的监控,对于保障食品安全、制定适 宜的限量标准是非常必要的。

伏马菌素的检测方法主要有薄层色谱法 (TLC)、气相色谱-质谱联用法 (GC-MS)、酶联免疫 吸附法 $(\text { ELISA })^{[13]}$ 、液相色谱-质谱联用法 ( LCMS $)^{[14]}$ 以及高效液相色谱法 ( HPLC $)^{[15,16]}$ 等。 HPLC 方法由于操作简单, 准确可靠, 重复性好, 是 目前国际上普遍采用的伏马菌素的检测方法 ${ }^{[16,17]}$ 由于伏马菌素分子没有特定的紫外吸收, 本身也不 产生苂光,应用 HPLC 时需要衍生后才能进行定性、 定量分析。目前 HPLC 以邻苯二甲醛 (o-phthalaldehyde, OPA) 柱前衍生为主 ${ }^{[16-18]}$, 操作简单, 反应 迅速, 但 OPA 衍生产物的荧光强度随着时间延长逐 渐降低, 对衍生反应时间要求相对较严格,需要在衍 生后 $2 \mathrm{~min}$ 内进样 ${ }^{[19]}$; 相比于柱前衍生, 柱后衍生 方法有效地克服了检测中苂光反应物稳定性对时间 的依赖,消除了基质效应以及杂质的干扰,具有稳定 性高、重现性好、操作简便、适用于大量样品的连续 检测等优势 ${ }^{[20]}$ 。利用 OPA 柱后衍生测定伏马菌素 方法虽有报道 ${ }^{[21]}$, 但缺乏对衍生 $\mathrm{pH}$ 值、衍生剂浓 度、衍生剂流速等多种柱后衍生影响因素的分析和 比较。本文优化了 OPA 柱后衍生的条件, 建立了玉
米中伏马菌素的柱后衍生-高效液相色谱方法, 实现 了伏马菌素的衍生反应和检测自动化控制, 避免了 人员操作的误差。结果表明,该方法准确、重现性 好,适用于批量样品的检测分析。

\section{1 实验部分}

\section{1 仪器与试剂}

Agilent 1200 高效液相色谱仪, 配苂光检测器 (Agilent 1200, 美国 Agilent 公司); PCX 柱后衍生 仪 (美国 Pickering Laboratories 公司); 高速万能粉 碎机 (天津市泰斯特仪器有限公司) ; IKA T18 组织 匀浆机 (广州仪科实验室技术有限公司), WH-90A 微型混合器 (上海亚荣生化仪器厂); Milli-Q 超纯 水仪 (美国 Millipore 公司); 固相萃取装置 (美国 Supelco 公司); SAX 固相萃取柱 (3 mL, $500 \mathrm{mg}$; 美国 Supelco 公司); DCY-III 水浴浓缩仪 (北京金 科精华苑技术研究所)；RE52-99 旋转蒸发器 (上海 亚荣生化仪器厂)。

$\mathrm{FB}_{1}$ 和 $\mathrm{FB}_{2}$ 标准品 (纯度 $>98 \%$, Sigma 公 司); OPA (Fluka 公司); $\beta$-颈基乙醇 (Sigma 公 司); 甲醇、乙腈 (HPLC 纯, 美国 Fisher 公司); 其他 试剂为分析纯 (北京化学试剂公司)。水为超纯水。

\section{2 衍生剂的配制}

OPA 稀释液: 称取 $6.11 \mathrm{~g}$ 四硼酸钾, 用 $100 \mathrm{~mL}$ $10 \mathrm{~g} / \mathrm{L}$ 氢氧化钾溶液溶解, 混匀即得。

OPA 衍生剂: 称取 $0.2 \mathrm{~g} \mathrm{OPA}$, 用 $5 \mathrm{~mL}$ 甲醇溶 解, 加人 $100 \mathrm{~mL} \mathrm{OPA}$ 稀释液, 混匀, 加人 $100 \mu \mathrm{L} \beta$ 颈基乙醇, 混匀,过 $0.2 \mu \mathrm{m}$ 滤膜。

\section{3 色谱条件}

色谱柱: ZORBAX SB-C ${ }_{18}$ 柱 $(150 \mathrm{~mm} \times 4.6$ $\mathrm{mm}, 5 \mu \mathrm{m}$; 美国 Agilent 公司); 流动相: $\mathrm{A}$ 相为 0.1 $\mathrm{mol} / \mathrm{L}$ 磷酸二氢钠溶液 (用磷酸调 $\mathrm{pH}$ 至 3.3 ) ; B 相为甲醇。梯度洗脱程序:0 2 $\mathrm{min}, 35 \% \mathrm{~A} ; 2 \sim 7$ $\min , 30 \% \mathrm{~A} ; 7 \sim 16 \mathrm{~min}, 20 \% \mathrm{~A} ; 16 \sim 18 \mathrm{~min}$,

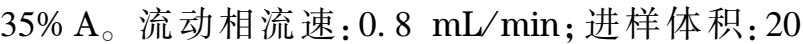
$\mu \mathrm{L}$; 柱温: $40{ }^{\circ} \mathrm{C}$ 。苂光检测器: 激发波长 $335 \mathrm{~nm}$, 发 射波长 $440 \mathrm{~nm}$ 。衍生反应线圈的体积为 $100 \mu \mathrm{L}$, 衍生温度为室温, $\mathrm{OPA}$ 衍生剂流速 $0.4 \mathrm{~mL} / \mathrm{min}$ 。

\section{4 样品处理方法}

实验所用玉米样品购自北京市集贸市场,用粉 碎机粉碎后,过 $0.45 \mathrm{~mm}$ 篮。

样品处理方法参考文献 ${ }^{[16]}$ 进行。称取 $50 \mathrm{~g}$ 试 样于 $150 \mathrm{~mL}$ 锥形瓶中, 准确加人 $100 \mathrm{~mL}$ 甲醇-水溶 液 $(3: 1, \mathrm{v} / \mathrm{v})$, 用高速匀浆机提取 $3 \mathrm{~min}$, 于 $4{ }^{\circ} \mathrm{C}$ 、 $5000 \mathrm{r} / \mathrm{min}$ 离心 $15 \mathrm{~min}$, 将上清液过滤至 $100 \mathrm{~mL}$ 
烧杯中。吸取 $10 \mathrm{~mL}$ 玉米样品提取液加人到预先 用 $5 \mathrm{~mL}$ 甲醇、 $5 \mathrm{~mL}$ 甲醇-水 $(3: 1, \mathrm{v} / \mathrm{v})$ 活化的 SAX 柱中, 待提取液通过 $\mathrm{SAX}$ 柱后, 依次用 $5 \mathrm{~mL}$ 甲醇水 $(3: 1, \mathrm{v} / \mathrm{v}) 、 3 \mathrm{~mL}$ 甲醇淋洗, 最后用 $10 \mathrm{~mL}$ 乙酸甲醇溶液 $(1: 99, \mathrm{v} / \mathrm{v})$ 进行洗脱, 收集洗脱液, 并于 $50{ }^{\circ} \mathrm{C}$ 水浴中旋转蒸发至近干, 取少量甲醇溶解残留 物并转移至样品瓶中, 再用少量甲醇分数次冲洗旋 转蒸发瓶, 同样转移至样品瓶中。将样品瓶置于 50 ${ }^{\circ} \mathrm{C}$ 水浴中通氮气吹干,除去洗脱液中的残留乙酸。吹 干后的样品在 $-18{ }^{\circ} \mathrm{C}$ 下保存,分析时用 $1 \mathrm{~mL}$ 的初始 流动相溶解,经 $0.2 \mu \mathrm{m}$ 滤膜过滤后,供 HPLC 分析。

\section{2 结果与讨论}

\section{1 衍生剂的 $\mathrm{pH}$ 对灵敏度的影响}

OPA 需在碱性条件下与硫醇和一级胺反应生 成具有苂光特性的物质, 缓冲液的 $\mathrm{pH}$ 值对反应的 灵敏性有一定的影响。为了确定合适的衍生剂的 $\mathrm{pH}$ 值,分别用四嗍酸钾和氢氧化钾溶液配制不同 $\mathrm{pH}$ 值的四硼酸钾缓冲液, 对 $2 \mathrm{mg} / \mathrm{L}$ 的 $\mathrm{FB}_{1}$ 标准溶 液进行测定。不同缓冲液 $\mathrm{pH}$ 对 $\mathrm{FB}_{1}$ 的峰面积的影 响见图 1。从图 1 可发现,随着缓冲液 $\mathrm{pH}$ 的升高, $\mathrm{FB}_{1}$ 衍生产物的峰面积逐渐增加, 当 $\mathrm{pH}$ 值大于 11 时, 峰面积开始下降, 且缓冲液的缓冲能力下降。为 了获得相对稳定的峰面积, 选择缓冲液的 $\mathrm{pH}$ 在 10.5 左右。经测定, 用 $10 \mathrm{~g} / \mathrm{L}$ 氢氧化钾溶液配制 $0.2 \mathrm{~mol} / \mathrm{L}$ 四嗍酸钾的 $\mathrm{pH}$ 在 10.5 左右, 能满足测 定的需要。

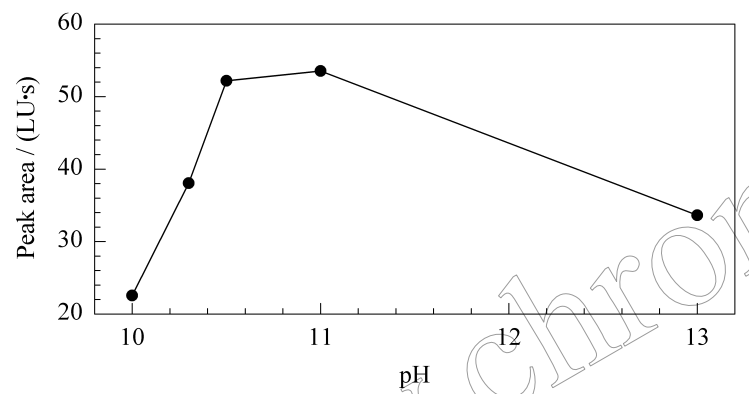

图 1 缓冲液 $\mathrm{pH}$ 值对 $\mathrm{FB}_{1}$ 衍生物峰面积的影响

Fig. 1 Effect of $\mathrm{pH}$ of derivatization buffer on peak area of $\mathbf{F B}_{1}$ derivative

In order to optimize the $\mathrm{pH}$ for OPA derivatization, adjust the buffer solution by potassium tetraborate and potassium hydroxide.

为了确定流动相 $\mathrm{A}$ 的 $\mathrm{pH}$ 对衍生效果的影响, 用磷酸将流动相 $\mathrm{A}$ 的 $\mathrm{pH}$ 分别调为 $3.0 、 3.3 、 3.5$ 、 4. 0 。结果发现, 随着流动相 $\mathrm{A} \mathrm{pH}$ 的提高, $\mathrm{FB}_{1}$ 和 $\mathrm{FB}_{2}$ 的保留时间逐渐缩短, 峰面积略有增加。然而, 随着 $\mathrm{pH}$ 增加, $\mathrm{FB}_{1}$ 与样品杂质峰的分离度降低。 因此,实验选择流动相 $\mathrm{A}$ 的 $\mathrm{pH}$ 为 3.3 。

\section{2 激发和发射波长的选择}

为了确定 $\mathrm{FB}_{1}$ 和 $\mathrm{FB}_{2}$ 衍生物的激发波长和发 射波长,分别对质量浓度为 $2 \mathrm{mg} / \mathrm{L}$ 的 $\mathrm{FB}_{1}$ 和 $\mathrm{FB}_{2}$ 标准溶液进行波长扫描。参考柱前衍生所采用的激 发波长和发射波长, 固定发射波长为 $440 \mathrm{~nm}$, 对激 发波长进行扫描,扫描范围为 $220 \sim 400 \mathrm{~nm}, \mathrm{FB}_{1}$ 、 $\mathrm{FB}_{2}$ 的激发波长扫描光谱图见图 2。从图 2 中可以 看出 $\mathrm{FB}_{1}$ 和 $\mathrm{FB}_{2}$ 的最大激发波长均在 $335 \mathrm{~nm}$ 。固
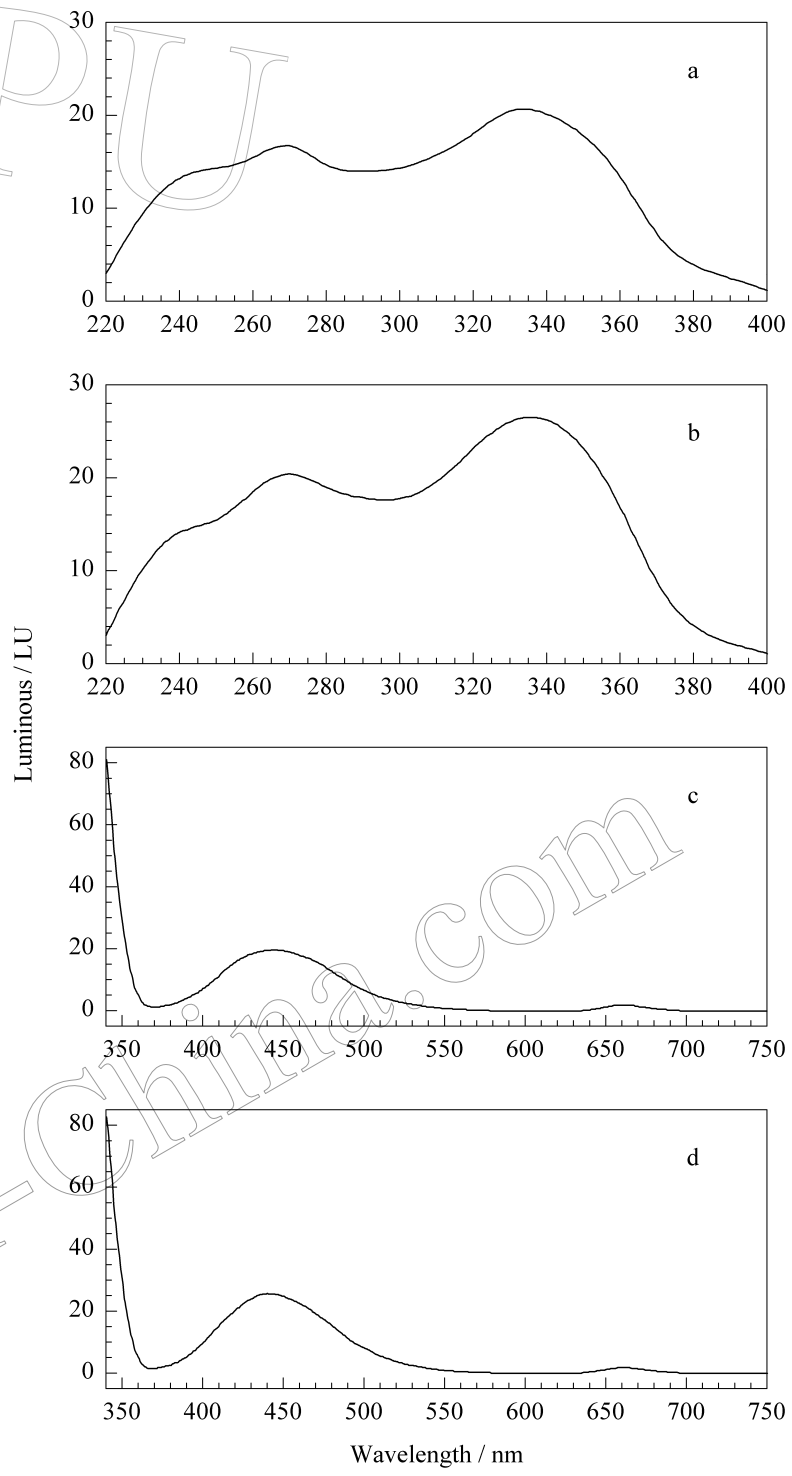

图 2 (a) $\mathrm{FB}_{1}$ 、( (b) $\mathrm{FB}_{2}$ 的激发波长扫描图和 (c) $\mathrm{FB}_{1}$ 、 (d) $\mathbf{F B}_{2}$ 的发射波长扫描图

Fig. 2 Excitation wavelength spectra of (a) $\mathbf{F B}_{1}$, (b) $\mathrm{FB}_{2}$ and emission wavelength spectra of (c) $\mathrm{FB}_{1}$, (d) $\mathrm{FB}_{2}$

The concentrations of $\mathrm{FB}_{1}$ and $\mathrm{FB}_{2}$ standard solution were 2 $\mathrm{mg} / \mathrm{L}$. Spectra of a and $\mathrm{b}$ were obtained by scanning the excitation wavelength scope from 220 to $400 \mathrm{~nm}$ under the condition of fixed emission wavelength of $440 \mathrm{~nm}$. Spectra of c and d were obtained by scanning the emission wavelength scope from 340 to $750 \mathrm{~nm}$ under the condition of fixed excitation wavelength of $335 \mathrm{~nm}$. 
定激发波长为 $335 \mathrm{~nm}$, 对发射波长进行扫描, 扫描 范围为 $340 \sim 750 \mathrm{~nm}, \mathrm{FB}_{1}$ 和 $\mathrm{FB}_{2}$ 的发射波长扫描 图见图 2。由图 2 中可以看出, $\mathrm{FB}_{1}$ 和 $\mathrm{FB}_{2}$ 的最大 发射波长为 $440 \mathrm{~nm}$ 。因此实验选择激发波长为 335 $\mathrm{nm}$, 发射波长为 $440 \mathrm{~nm}$ 。

\subsection{OPA 浓度对测定结果的影响}

称取适量的 OPA 用甲醇溶解, 加人到 OPA 缓 冲体系中, 使衍生剂中 $\mathrm{OPA}$ 的质量浓度分别为 $0.5 、 1.0 、 2.0 、 3.0 、 4.5 \mathrm{~g} / \mathrm{L}$, 对质量浓度为 $2 \mathrm{mg} / \mathrm{L}$ 的 $\mathrm{FB}_{1}$ 和 $\mathrm{FB}_{2}$ 混合标准溶液进行色谱分析,结果见 图 3。从图 3 中可以看出, 随着 $\mathrm{OPA}$ 浓度的增加, 伏马菌素衍生物的峰面积逐渐增加, 从 0.5 到 2.0 $\mathrm{g} / \mathrm{L}$ 时增加效果非常显著; 继续增加 OPA 浓度, 峰 面积增加速度相对缓慢。同时发现 OPA 浓度升高 会导致基线升高,因此本方法选择 OPA 的质量浓度 为 $2 \mathrm{~g} / \mathrm{L}$ 。

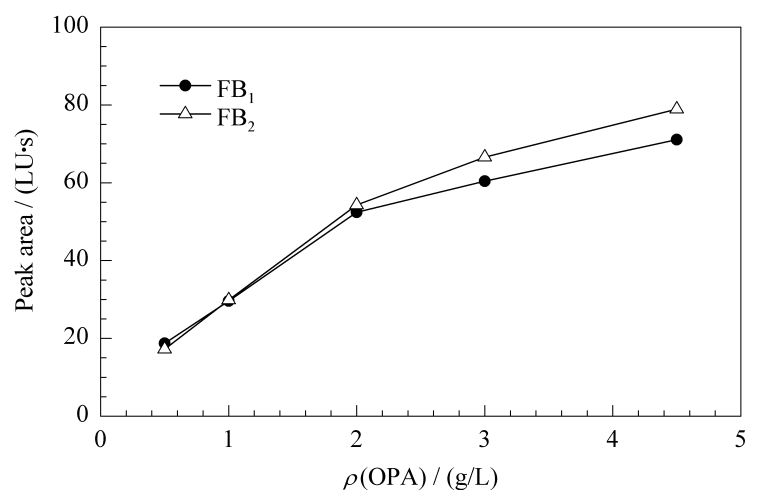

图 $30 P A$ 浓度对 $\mathrm{FB}_{1} 、 \mathrm{FB}_{2}$ 峰面积的影响

Fig. 3 Effect of OPA concentration on peak areas of $\mathrm{FB}_{1}, \mathrm{FB}_{2}$

Conditions: $\mathrm{pH}$ of derivatization buffer, 10.5; flow rate of OPA, $0.4 \mathrm{~mL} / \mathrm{min}$.

\section{4 衍生剂的流速对衍生反应的影响}

柱后衍生的效果与衍生剂的流速关系密切。为 了获得较好的衍生效果, 将流动相的流速设为 0.8 $\mathrm{mL} / \mathrm{min}$,调整衍生剂的流速分别为 $0.3 、 0.4 、 0.5$ 、 $0.6 \mathrm{~mL} / \mathrm{min}$, 对质量浓度为 $2 \mathrm{mg} / \mathrm{L}$ 的 $\mathrm{FB}_{1}$ 和 $\mathrm{FB}_{2}$ 混合标准溶液进行色谱分析,结果见图 4。从图 4 中可以看出, 随着衍生剂流速的增加, $\mathrm{FB}_{1}$ 和 $\mathrm{FB}_{2}$ 峰面积逐渐提高。这可能是由于衍生剂在低流速 时,单位时间内与样品中伏马菌素发生反应的 OPA 数量较少,生成的苂光产物相对较少, 苂光强度较 低; 随着衍生剂流速的增加, 单位时间内与样品反应 的 OPA 数量增加, 峰面积也随之增加。然而, 随着 流速的增加,一方面使衍生体系洜的压力会逐渐增 加, 噪声提高; 另一方面会造成衍生剂的浪费。因 此, 实验选择衍生剂的流速为 $0.4 \mathrm{~mL} / \mathrm{min}$ 。

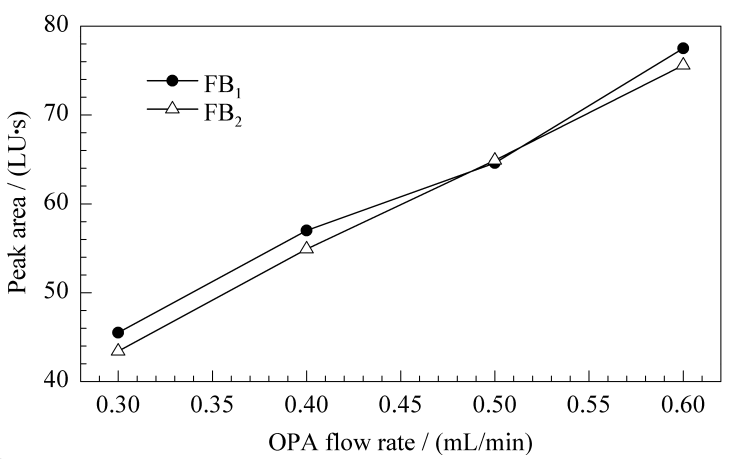

图 4 衍生剂的流速对 $\mathrm{FB}_{1} 、 \mathrm{FB}_{2}$ 峰面积的影响

Fig. 4 Effect of flow rate of OPA on peak areas of $\mathrm{FB}_{1}$ and $\mathrm{FB}_{2}$

Conditions: $\mathrm{pH}$ of derivatization buffer, 10.5 ; mass concentration of OPA, $2 \mathrm{~g} / \mathrm{L}$.

\section{5 不同溶剂对分离效果的影响}

分别用甲醇、乙腈-水 $(1 / 1, \mathrm{v} / \mathrm{v})$ 和初始流动相 配制 $1 \mathrm{mg} / \mathrm{L}$ 的 $\mathrm{FB}_{1}$ 和 $\mathrm{FB}_{2}$ 混合标准溶液进行色谱 分析,不同溶剂对伏马菌素分离效果的影响见图 5。 从图 5 可以看出,不同溶剂对 $\mathrm{FB}_{1}$ 和 $\mathrm{FB}_{2}$ 的分离 度、响应值有一定的影响, 尤其对 $\mathrm{FB}_{1}$ 的影响较大。 以流动相作为溶剂时, 色谱分离效果好, 响应值最 强; 以甲醇为溶剂时响应值最低。因此实验选择用 初始流动相溶解标准溶液和样品。标准溶液和玉米 样品的色谱图见图 6 。

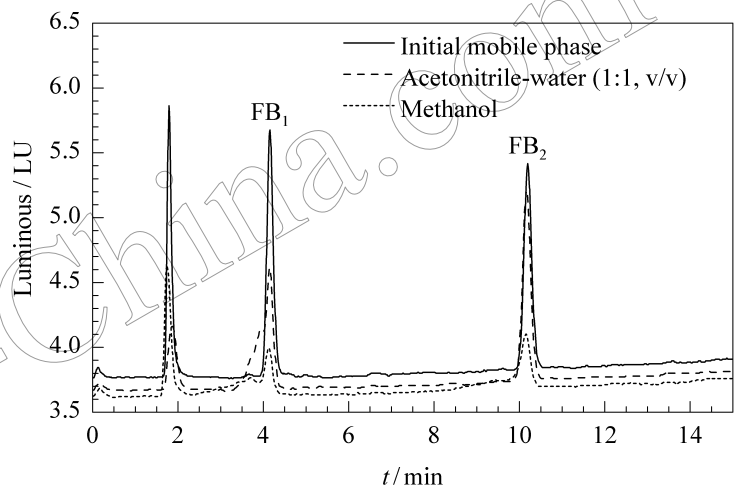

图 5 不同溶剂溶解样品对 $F_{1} 、 F_{2}$ 分离效果的影响

Fig. 5 Effect of different solvents for $\mathrm{FB}_{1}$ and $\mathrm{FB}_{2}$ separation

\section{6 线性范围、检出限、准确度和精密度}

分别测定用初始流动相配制的质量浓度为 $0.2 、 0.5 、 1.0 、 2.0 、 5.0 、 10 、 20 \mathrm{mg} / \mathrm{L}$ 的 $\mathrm{FB}_{1} 、 \mathrm{FB}_{2}$ 混 合标准溶液, 以各分析物的质量浓度 $(x)$ 为横坐标、 色谱峰面积 $(y)$ 为纵坐标进行回归分析, 得到的 $\mathrm{FB}_{1}$ 的线性方程为 $y=25.153 x-1.7061$, 相关系数 为 $0.9999 ; \mathrm{FB}_{2}$ 的线性方程为 $y=25.882 x-$ 0.4238 , 相关系数为 0.9998 。以信噪比 $(S / N)$ 为 3 求得 $\mathrm{FB}_{1} 、 \mathrm{FB}_{2}$ 的检出限均为 $0.02 \mathrm{mg} / \mathrm{kg}$ 。 


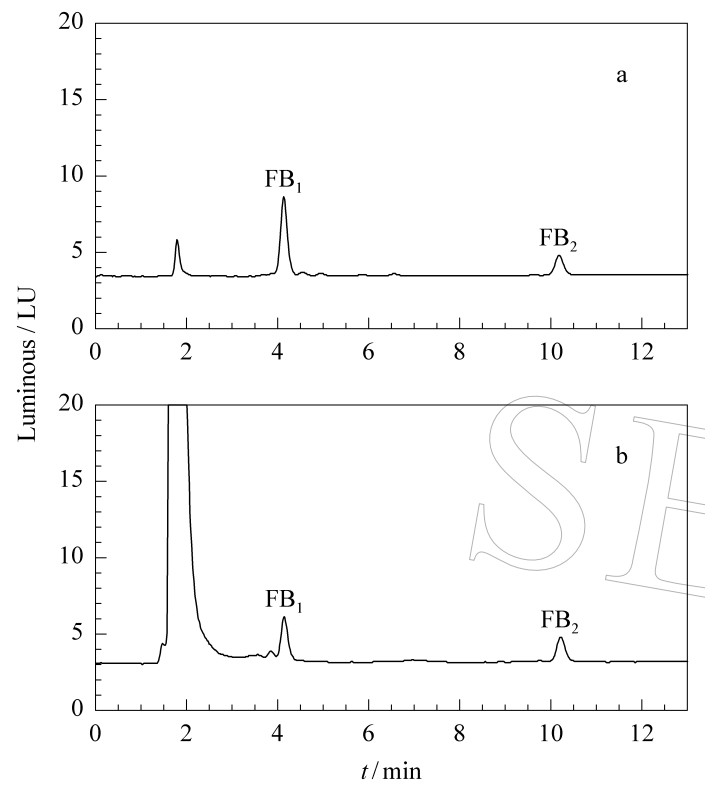

图 6 (a) 标准溶液和 (b) 玉米样品的色谱图

Fig. 6 Chromatograms of (a) standard solution and (b) a corn sample

将玉米样品分成 2 份, 1 份中不添加 $\mathrm{FB}_{1} 、 \mathrm{FB}_{2}$ 作为空白样品测定; 1 份中分别添加 $0.1 、 1.0 、 4.0$ $\mathrm{mg} / \mathrm{kg}$ 的 $\mathrm{FB}_{1}$ 和 $0.1 、 0.5 、 2.0 \mathrm{mg} / \mathrm{kg}$ 的 $\mathrm{FB}_{2}$, 按样 品处理方法提取、分析。玉米样品中伏马菌素的加 标回收率和精密度 (以相对标准偏差 (RSD) 计) 测 定结果见表 1 。结果显示, $\mathrm{FB}_{1}$ 的回收率为 $82.5 \%$ $\sim 88.0 \%, \mathrm{RSD}$ 为 $3.6 \% \sim 5.3 \% ; \mathrm{FB}_{2}$ 的回收率为 $83.0 \% \sim 89.8 \%, \mathrm{RSD}$ 为 $3.2 \% \sim 5.6 \%$ 。

\section{表 1 玉米样品中伏马菌素的加标回收率和精密度}

Table 1 Recoveries and precisions (RSDs) of fumonisins spiked in a corn sample $(n=6)$

\begin{tabular}{cccccc}
\hline Compound & $\begin{array}{c}\text { Background/ } \\
(\mathrm{mg} / \mathrm{kg})\end{array}$ & $\begin{array}{c}\text { Spiked/ } \\
(\mathrm{mg} / \mathrm{kg})\end{array}$ & $\begin{array}{c}\text { Found/ } \\
(\mathrm{mg} / \mathrm{kg})\end{array}$ & $\begin{array}{c}\text { Recovery/ } \\
\%\end{array}$ & $\begin{array}{c}\mathrm{RSD} / \\
\%\end{array}$ \\
\hline $\mathrm{FB}_{1}$ & nd & 0.1 & 0.0825 & 82.5 & 3.9 \\
& nd & 1.0 & 0.837 & 83.7 & 3.6 \\
$\mathrm{FB}_{2}$ & nd & 4.0 & 3.52 & 88.0 & 5.3 \\
& nd & 0.1 & 0.0830 & 83.0 & 3.2 \\
& nd & 0.5 & 0.449 & 89.8 & 3.5 \\
& nd & 2.0 & 1.72 & 86.0 & 5.6 \\
\hline
\end{tabular}

nd: not detected.

\section{7 玉米样品分析}

按照上述优化方法测定了 10 个玉米样品, 结果 见表 2 。通过检测发现, 10 个样品中有 7 个样品含 有伏马菌素, 阳性率为 $70 \%$; 从组成上看,玉米中 $\mathrm{FB}_{1}$ 的含量高于 $\mathrm{FB}_{2}$, 这与 Rheeder 等 ${ }^{[2]}$ 的报道相 符合。他们认为在玉米或大米的培养物中 $\mathrm{FB}_{1}$ 占 整个伏马菌素的 $70 \% \sim 80 \%, \mathrm{FB}_{2}$ 为 $15 \% \sim 25 \%$ 。从 含量上看, 有 3 个样品的含量超过了 $1 \mathrm{mg} / \mathrm{kg}$, 其中 1 个样品中的含量达到了 $4.09 \mathrm{mg} / \mathrm{kg}^{\circ}$ 。上述结果
说明,本方法可以用于玉米中伏马菌素的检测, 同时 也提示我国玉米中伏马菌素的安全性不容乐观, 加 强对玉米中伏马菌素的监控对保护消费者健康具有 重要的意义。

表 210 个玉米样品中 $\mathrm{FB}_{1}$ 和 $\mathrm{FB}_{2}$ 的含量

Table 2 Contents of $\mathrm{FB}_{1}$ and $\mathrm{FB}_{2}$ in 10 corn samples

\begin{tabular}{cccc}
\hline Sample & $\begin{array}{c}\text { Content of } \\
\mathrm{FB}_{1} / \\
(\mathrm{mg} / \mathrm{kg})\end{array}$ & $\begin{array}{c}\text { Content of } \\
\mathrm{FB}_{2} / \\
(\mathrm{mg} / \mathrm{kg})\end{array}$ & $\begin{array}{c}\text { Total content of } \\
\mathrm{FB}_{1} \text { and } \mathrm{FB}_{2} / \\
(\mathrm{mg} / \mathrm{kg})\end{array}$ \\
\hline 1 & 3.27 & 0.821 & 4.09 \\
2 & 0.0633 & $\mathrm{nd}$ & 0.0633 \\
3 & nd & $\mathrm{nd}$ & $\mathrm{nd}$ \\
4 & 1.42 & 0.205 & 1.62 \\
5 & nd & nd & nd \\
6 & 0.314 & 0.0468 & 0.361 \\
7 & 0.526 & 0.107 & 0.633 \\
8 & 0.285 & 0.0659 & 0.351 \\
9 & 0.291 & 0.779 & 1.07 \\
10 & nd & nd & nd \\
\hline Mean & 0.0617 & 0.202 & 0.819 \\
\hline
\end{tabular}

nd: not detected.

\section{3 结论}

本实验建立了 OPA 柱后衍生-高效液相色谱测 定玉米中 $\mathrm{FB}_{1} 、 \mathrm{FB}_{2}$ 的方法。与柱前衍生相比,柱后 衍生反应由衍生装置控制, 有效地保证了衍生时间 的精确控制, 消除了柱前衍生法普遍存在的受人为 因素影响的弊端,具有稳定性高、重现性好、操作简 便、适用于大量样品连续检测等优势, 为食品中伏马 菌素的检测提供了新的方法和思路。

\section{参考文献:}

[1] Gelderblom W C A, Jaskiewicz K, Marasas W F O, et al. J Appl Environ Microbiol, 1988, 54(7) : 1806

[2] Rheeder J P, Marasas W F O, Vismer H F. J Appl Environ Microbiol, 2002, 68(5) : 2101

[3 ] Ross P F, Ledet A E, Owens D L, et al. J Vet Diagn Invest, $1993,5(1): 69$

[4] Milićević D R, Škrinjar M, Baltić T. Toxins, 2010, 2 (4) : 572

[5] Voss K A, Smith G W, Haschek W M. Anim Feed Sci Tech, 2007, 137(3): 299

[6] Bouhet S, Oswald I P. Mol Nutr Food RES, 2007, 51 (8): 925

[7] Odhav B, Adam J K, Bhoola K D. Int Immunopharmacol, 2008, 8(6): 799

[8] Stockmann-Juvala H, Savolainen K. Hum Exp Toxicol, 2008, 27(11): 799

[9] Yazar S, Omurtag G Z. Int J Mol Sci, 2008, 9(11) : 2062

[10] Munkvold G P, Desjardins A E. Plant Dis, 1997, 81 (6): 556

[11] Food and Agriculture Organization of the United Nations and World Health Organization. Fifty-sixth Report of the 
Joint FAO/WHO Expert Committee on Food Additives. [2012-03-26]. http://www. who. int/foodsafety/chem/jec$\mathrm{fa} /$ publications/reports/en/index. html

[12] Commission Regulation ( EC) No. 1126/2007. Off J Eur Union, 2007, 50 ( L255) : 14

[13] Quan W Y, Gu J. Chinese Journal of Health Laboratory Technology (权伍英，谷晶. 中国卫生检验杂志)，2010，20 (4) : 948

14 Dall' Asta C, Galaverna G, Aureli G, et al. World Mycotoxin $\mathrm{J}, 2008,1(3): 237$

[15] Silva L, Fernández-Franzón M, Font G, et al. Food
Chem, 2009, 112(4) : 1031

[16] BS EN 13585-2002

[17] SN/T 1572-2005

[18 ] De Girolamo A, Fauw D P, Sizoo E, et al. World Mycotoxin J, 2010, 3(2) : 135

[19] Sydenham E W, Shephard G S, Thiel P G. J Assoc Off Anal Chem, 1992, 75(2): 313

[20 ] Muscarella M, Magro S L, Nardiello D, et al. J Chromatogr A, 2008, 1203(1): 88

[21 ] Akiyama H, Uraroongroj M, Miyahara M, et al. Mycopathologla , 1997, 140(3): 157

\section{3 年《岩矿测试》征订启事}

\section{国内统一刊号：CN11-2131/TD}

国际 CODEN： YACEEK

国外发行代号： BM4089
国际标准刊号：ISSN 0254-5357

国内邮发代号: 2-313 京西工商广字第 0227 号

《岩矿测试》1982 年创刊, 是中国地质学会岩矿测试技术专业委员会和国家地质实验测试中心共同主办 的学术期刊。是中国唯一的地质分析测试专业期刊, 曾获国家级优秀科技期刊三等奖, 地质矿产部优秀科技 期刊一等奖,北京市科技期刊四通杯全优期刊奖,中国科协优秀学术期刊三等奖。

《岩矿测试》是中文核心期刊 ( “地质学” 类)，中国科技核心期刊, 中国期刊方阵双效期刊。2010 年度的 影响因子为 1.280 , 在 1998 种中国科技核心期刊中排名第 61 位, 在 32 种地质科学类期刊中排名第 8 位。被 美国《化学文摘》、俄罗斯《文摘杂志》、美国《剑桥科学文摘》、美国《乌利希期刊指南》、英国《皇家化学学 会》系列文摘的《分析文摘》和《质谱学通报 - 增补》、波兰《哥白尼索引》, 《中国科学引文数据库》(CSCD)、 《中国期刊网》(CNKI)、《中文科技期刊全文数据库》、《万方数据一一科技化期刊群》等 18 家国内外数据库 和文摘收录。

《岩矿测试》以国家需求为导向, 坚持地质实验技术创新、面向应用、服务基层的方针和基本定位。以发 表优秀的地质与地球化学分析研究成果为核心目标, 报道国内外地质科学、环境保护、石油化工、冶金、煤炭 及其相关领域的基础性、前瞻性和创新性研究成果, 关注地学领域中的热点问题及边缘学科, 促进我国分析 测试技术的发展。注重学术的参考价值, 追求技术创新方法实用, 研究思路和写作内涵能够给读者启迪与借 鉴。本刊适合于地质、冶金、环保、石油、化工、煤炭等行业从事分析测试的科技工作者及大专院校师生阅读。

《岩矿测试》远程稿件采编系统 (http://www. ykcs. ac. cn), 实现了稿件采编自动化,信息发布实时化, 过刊全文可免费下载。

《岩矿测试》为双月刊, 大 16 开, 逢双月 15 日出版; 国内外公开发行。2013 年定价 30.0 元/本, 全年 180.00 元。漏订的读者可直接与编辑部联系。

\section{《岩矿测试》编辑部}

通讯地址: 北京市西城区百万庄大街 26 号 国家地质实验测试中心 (邮政编码 100037)

电话: (010)68999562

传真: (010)68999563

E-mail: ykcs_zazhi@163.com; ykcs_zazhi@sina.com 